УДК 621.791.052.8:622.692

ПОВЫШЕНИЕ ЭФФЕКТИВНОСТИ ВХОДНОГО КОНТРОЛЯ

СВАРЩИКОВ ПРИ СТРОИТЕЛЬСТВЕ ОПАСНЫХ ПРОИЗВОДСТВЕННЫХ ОБЬЕКТОВ

\title{
INCREASING THE EFFICIENCY OF INPUT INSPECTION OF WELDERS IN THE CONSTRUCTION OF HAZARDOUS PRODUCTION FACILITIES
}

\author{
А.М. Файрушин, И.А. Марченко, В.С. Хазиев
}

Уфимский государственный нефтяной технический университет, г. Уфа, Российская Федерация

Airat M. Fairushin, Ilya A. Marchenko, Viktor S. Khaziev

Ufa State Petroleum Technological University, Ufa, Russian Federation e-mail: otsk@ rusoil.net

Аннотация. Сварка является одним из ведущих технологических процессов обработки металлов. Сварочные технологии широко применяются при строительстве опасных производственных объектов нефтегазового комплекса. Требования к организации сварочных работ на опасных производственных объектах прописаны отдельным разделом в Федеральных нормах и правилах в области промышленной безопасности.

Оценка квалификации сварщика после его обучения, а также при приеме его на работу и прохождении переаттестации всегда носит субъективный характер, так как специалист, проверяющий контрольное сварное соединение, ориентируется в основном на наличие или отсутствие дефектов и не дает характеристику выполненного шва относительно какого-либо эталона. 
В последние несколько лет прослеживаются тенденции роста сдачи практического испытания в онлайн-режиме, что позволяет комиссии или работодателю оценивать квалификацию сварщиков дистанционно. Однако, как показывает практика, нет единого подхода к процедуре проведения тестирования, и на сегодняшний день встает вопрос о выработке предложений для эффективной организации процедуры оценки квалификации сварщика с использованием современных средств технического оснащения.

Оценка квалификации сварщика на современном этапе основывается на применении различных методов неразрушающего контроля сварных соединений. Неразрушающие методы контроля, такие как визуальноизмерительный контроль, ультразвуковой или рентгенографический, весьма сложные, дорогостоящие и не в полной мере определяют квалификационный уровень сварщика, а только дают информацию о качестве сварного шва по принципу - соответствует или не соответствует требованиям и зависят от норм дефектности для различных отраслей промышленности.

Оценка качества выполненных контрольных сварных соединений с применением технологии 3DLD дает максимально объективную характеристику уровня подготовки сварщика и его возможности в профессиональном плане.

Abstract. Welding is one of the leading technological processes in metal processing. Welding technologies are widely used in the construction of hazardous production facilities in the oil and gas complex. Requirements for the organization of welding at hazardous production facilities are spelled out in a separate section in the Federal codes and regulations in the field of industrial safety.

Evaluation of the qualification of the welder after his training, as well as when hiring him and undergoing re-certification is always subjective, since the specialist who checks the control welded joint is guided mainly by the presence 
or absence of defects and does not give a characteristic of the weld made relative to any standard.

In the past few years, there have been trends in the growth of online practical testing, which allows the commission or the employer to assess the qualifications of welders remotely. However, as practice shows, there is no single approach to the testing procedure and today the question arises of developing proposals for the effective organization of the welder qualification assessment procedure using modern technical equipment.

Assessment of the qualifications of a welder at the present stage is based on the use of various methods of non-destructive testing of welded joints. Nondestructive testing methods, such as visual inspection, ultrasonic or X-ray, are very complex, expensive and do not fully determine the qualification level of the welder, but only give information about the quality of the weld according to the principle meets or does not meet the requirements, and depend on the standards of defectiveness, for various industries.

Evaluation of the quality of the performed control welded joints using 3DLD technology gives the most objective characteristic of the welder's training level and his professional capabilities.

Ключевые слова: видеофиксация; сварщик; сварочный полигон; контроль сварки; оценка квалификации сварщика; контрольные сварные соединения

Key words: video fixation; welder; welding site; welding control; qualification assessment of welder; control welded joints

Сварка считается важнейшем процессом в производстве, которому уделяется большое внимание во всем мире и в России. В Федеральных нормах и правилах в области промышленной безопасности «Правила промышленной безопасности опасных производственных объектов, на которых используется оборудование, работающее под избыточным 
давлением» вопросы сварочного производства отражены в специальном разделе «Сварка».

Основное направление развития сварочных технологий сегодня - это автоматизация производства с целью снижения трудоемкости, повышения качества и производительности труда. В связи с этим видеонаблюдение сварочного процесса становится все более актуальным. Видеонаблюдение в сфере сдачи практических экзаменов у сварщиков применяются уже более 5 лет, но требования к ним на сегодняшний день не определены.

Оценка квалификации сварщика на сегодняшний день ориентируется в основном на наличие или отсутствие дефектов в контрольном сварном соединении. Однако не менее важно, по мнению многих известных ученых, оценивать квалификацию конкретного сварщика на основе анализа стабильности его моторных действий в процессе формирования шва [1].

Многие учебные заведения при подготовке сварщиков приняли обязательства по проведению Государственной итоговой аттестации (ГИА) в соответствии с технологиями WorldSkills и проведении практического демоэкзамена выпускника, который фиксируется с помощью видеокамер.

В настоящее время подтверждение квалификации, как правило, осуществляется при проведении практического испытания на соответствующем полигоне (посту). Требования к таким постам имеют общий характер: сварочный пост, стационарный, размером 2,0 × 2,5 м, оснащённый столом для проведения сварки, сварочным аппаратом и вентиляцией. На данный момент точно не указаны требования к системе видеофиксации, и что именно она должна фиксировать. В интернете можно найти большое количество видеоматериалов, регистрирующих работу сварщика, на которой возможно идентифицировать сварщика, но практически невозможно понять, какая у него квалификация.

Например, на рисунке 1 изображен кадр из видеоматериала демонстрационного экзамена в одном из сварочных центров. Кадр сделан с видеокамеры, установленной на полигоне. В данный момент на полигоне 
проходит практическую аттестацию сварщик, и мы видим, что данная камера не показывает те требования, которые необходимы для оценки квалификации сварщика при проведении экзамена.

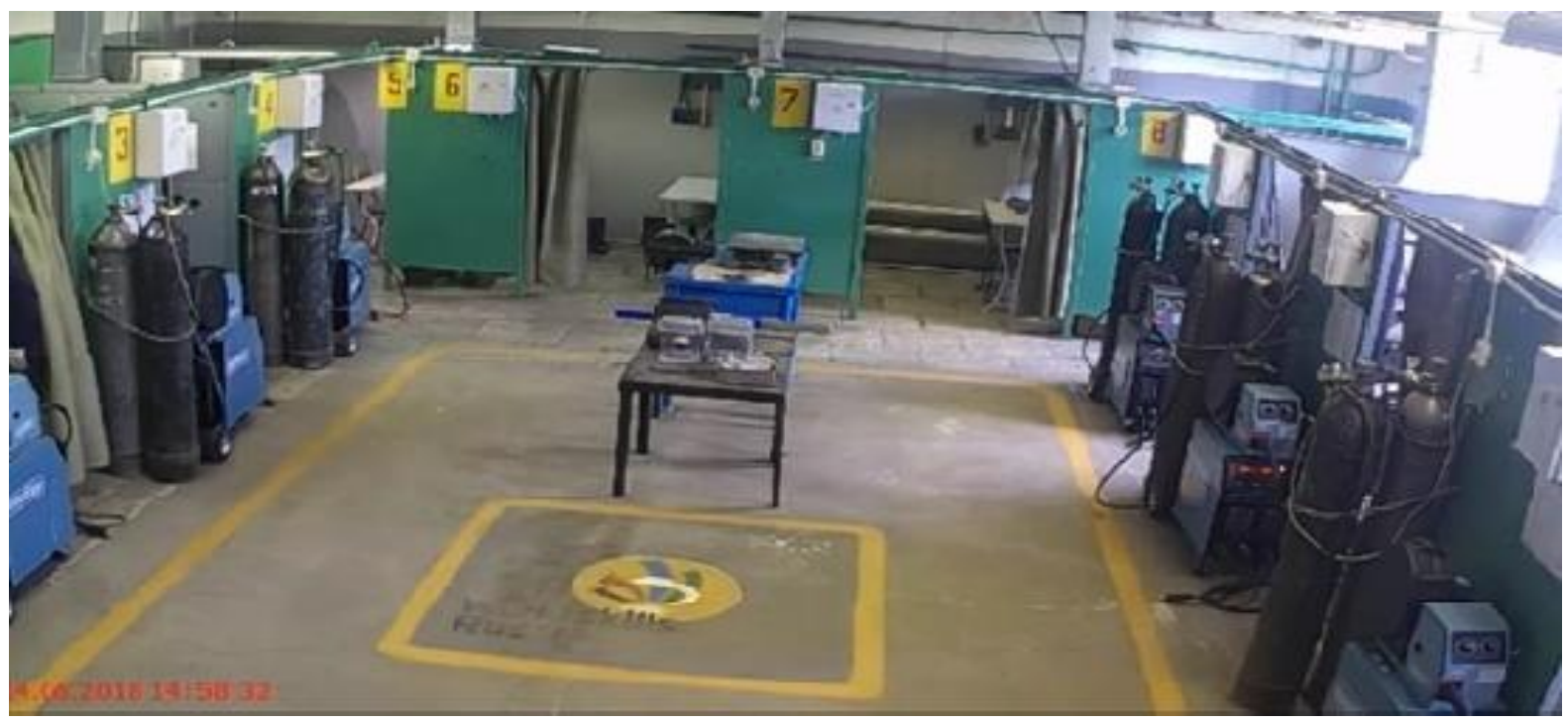

Рисунок 1. Кадр из видеоматериала с практического испытания сварщика

В других видеоматериалах (рисунок 2) можно увидеть, что камера установлена на посту, но она также не дает возможности наблюдателю понять степень владения сварщиком требуемыми навыками.

Идея решения в том, что система видеонаблюдения должна быть установлена так, чтобы наблюдатель видел не только тестируемого, но также мог понять квалификацию сварщика. Это возможно в том случае, если камера стоит на посту и фиксирует не только работу сварщика, но и панель приборов сварочного аппарата с возможностью наблюдения за изменением характеристик сварочного тока.

Как правило, если сварщик имеет хорошую квалификацию, то в процессе сварки он стабильно держит дугу, и сила сварочного тока в процессе сварки колеблется в пределах не более \pm 10 А. 


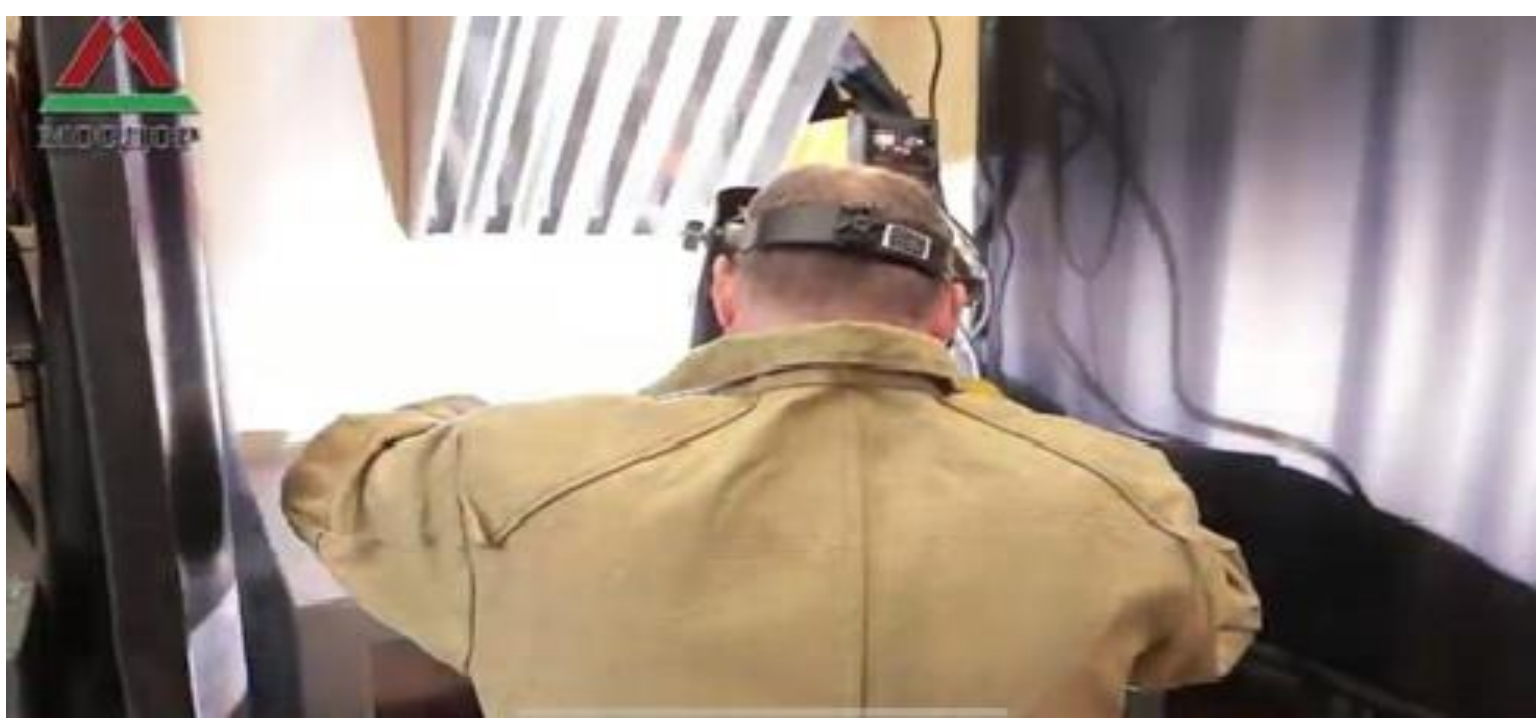

Рисунок 2. Кадр и видеоматериала с камеры, установленной на посту [2]

На рисунке 3 камера настроена так, что можно определить, в каком положении и какие детали варит сварщик, а также характеристики изменения режима сварки, фиксируемые на панели приборов сварочного аппарата (выделены красным овалом), которые дают понимание о том, как экзаменуемый ведет процесс сварки, стабильность и плавность манипуляций, скорость сварки, т.е. возможно дистанционно оценивать моторные действия сварщика, определяющие его квалификацию.

В связи с тем, что не все модели сварочных аппаратов имеют информативные панели, для организации видеофиксации режимов сварки можно установить специальные системы регистрации параметров сварочных процессов.

Несмотря на то, что наблюдение за процессом выполнения сварки дает хорошую информацию о квалификации сварщика, конечным результатом его работы является бездефектный сварной шов. В связи с этим необходимо в дальнейшем провести визуально-измерительный контроль сварного соединения с целью оценки квалификации сварщика по качеству выполнения сварного шва. 


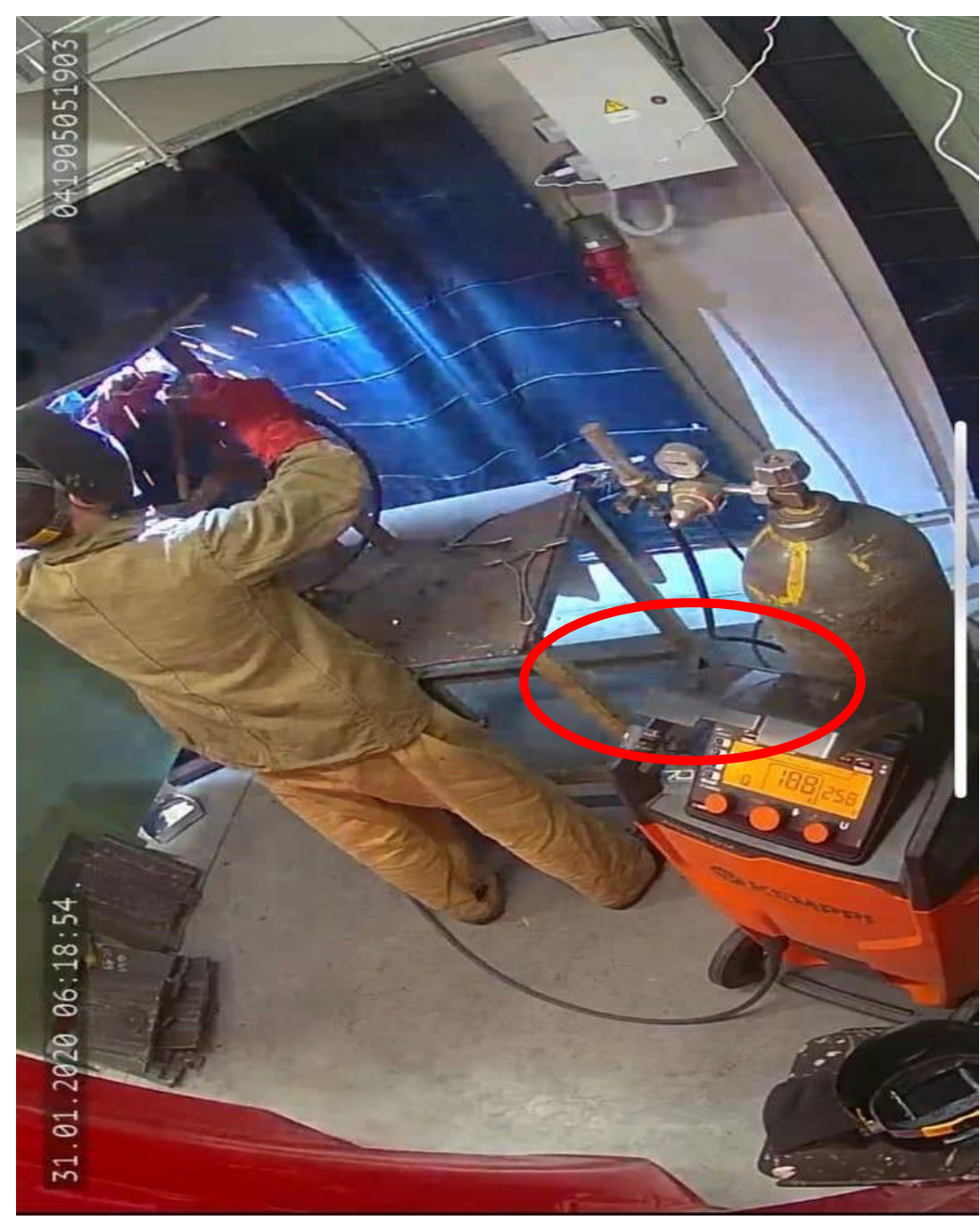

Рисунок 3. Кадр из видеоматериала с поста с предлагаемой системой видеофиксации

Для оценки квалификации сварщика мы предлагаем использовать метод оценки по технологии 3DLD, основанный на принципе сравнения сварного шва с расчетным эталоном без дефектов [3-9].

Оценка квалификации сварщика выполняется с применением лазерной диагностики формы поверхности сварного шва, которая работает по принципу сравнения расчетной (эталонной) формы лицевой и корневой поверхности сварного шва с формой цифровых реплик лицевой и корневой поверхности сварного шва, выполненного сварщиком, и идентифицирует практические навыки сварщика цифровым индексом квалификации сварщика (ИКС).

Сканирование контрольного сварного соединения происходит автоматически с высокой степенью разрешения - на длине 250 мм 
проводится замер 1000 сечений сварного шва, что дает возможность с высокой точностью выявлять все несоответствия по длине шва (рисунок 4).

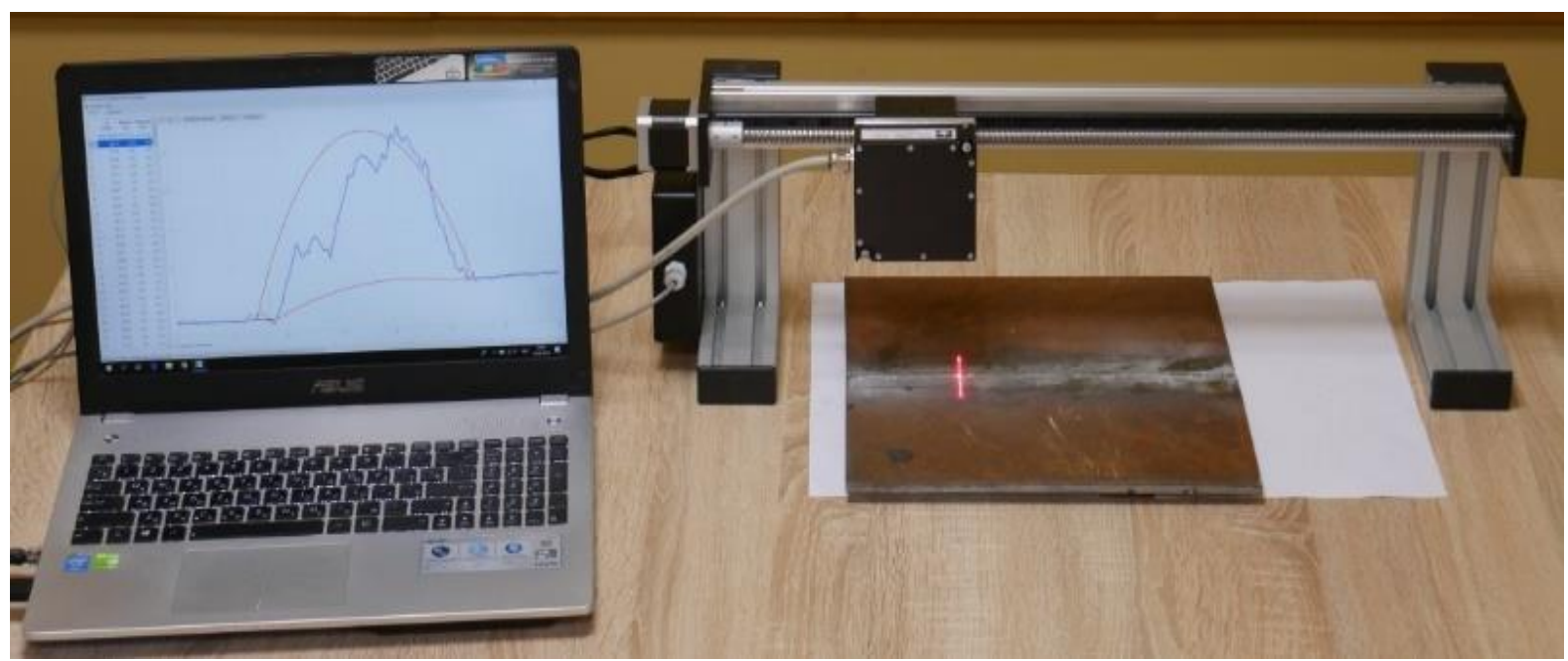

Рисунок 4. Фото сканирования контрольного сварного образца

После окончания сканирования программа автоматически формирует цифровую реплику лицевой и корневой поверхности сварного шва, выполненной сварщиком. Данные сканирования 3DLD сохраняются в электронном виде для каждого сварщика (рисунок 5).

Программа определяет индекс квалификации сварщика и визуализирует отклонения формы поверхности сварного шва от расчетного эталона, окрашивая их разными цветами. На рисунке 5 эталонная форма шва изображена красной сеткой.

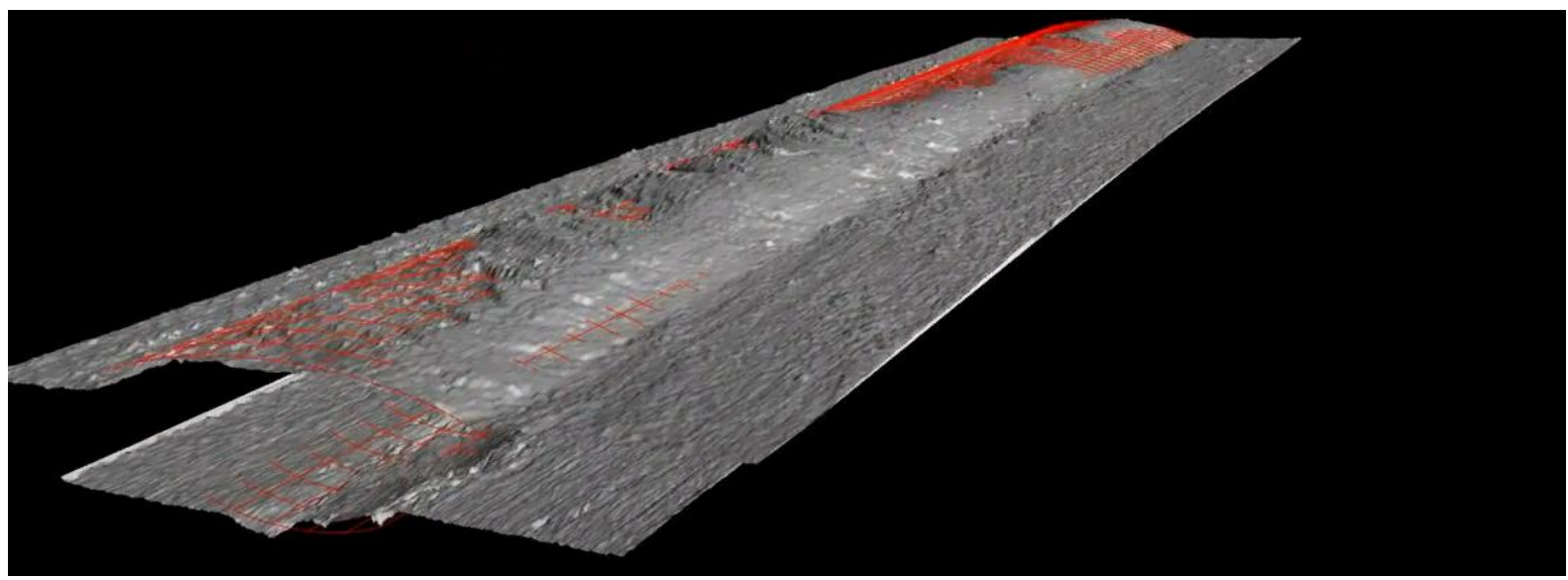

Рисунок 5. Цифровая реплика сварного шва (вверху - лицевая поверхность сварного соединения; внизу - корень шва) 
Определение индекса квалификации сварщика выполняется с применением результатов сканирования по отклонению поверхности сварного шва от эталона по формуле [5]:

$$
Q W=\frac{100}{N} \sum_{j=1}^{N}\left(1-\frac{S_{j}^{\text {откл }}}{S^{\ni}}\right)^{\prime}
$$

где $Q W$ - индекс квалификации сварщика (ИКС);

$N=L w / d L w+1-$ количество измеренных сечений сварного шва с шагом измерения $d L w$ большим или равным 0,1 мм;

$L w$ - длина сварного шва;

$j$ - номер текущего значения, измеренного поперечного сечения сварного шва;

$S^{\ni}$ - площадь эталона;

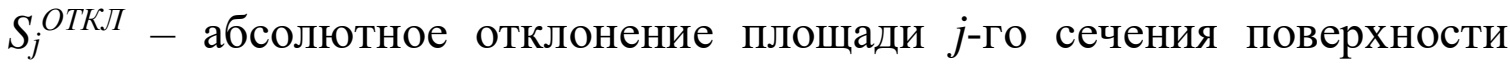
сварного шва от площади эталона, которое определяется по формуле:

$$
S_{j}^{\text {оТкл }}=\int_{0}^{B} Z_{j}^{\text {ОТКл }}(x) d x,
$$

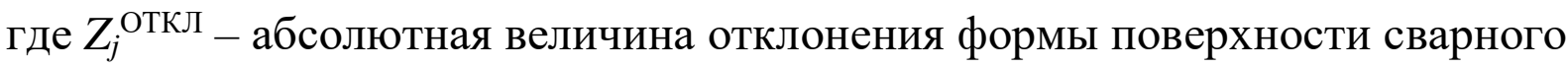
шва в $j$-ом сечении от внешней и внутренней формы эталона, которая определяется по формуле:

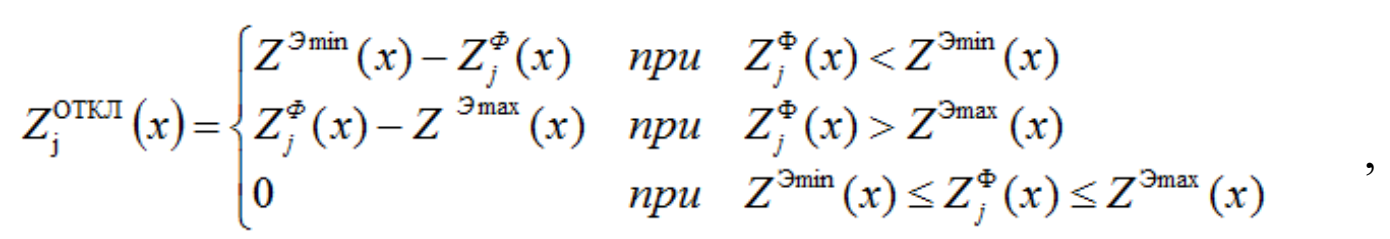

где $B$ - ширина сканирования. Значения $B$ находятся в интервале от $1,5 e_{\max }$ до $3 e_{\max }$;

$e_{\max }$ - максимальная ширина сварного шва, устанавливается нормативнотехническими документами или требованиями Заявителя;

$Z^{\Phi}{ }_{j}(x)$ - измеренные значения координат поверхности $j$-го поперечного сечения сварного шва с шагом $d x$ от 0,1 мм до 1 мм;

$Z, x$ - координаты в системе координат ZOX; 
$Z^{\text {эmax }}(x)$ - кривая, определяющая внешнюю форму эталона сварного шва;

$Z^{\text {Эmin }}(x)$ - кривая, определяющая внутреннюю форму эталона сварного шва.

Внешнюю $Z^{\ni \max }(x)$ и внутреннюю $Z^{\ni \min }(x)$ формы эталонных кривых сварного шва в любом пространственном положении вычисляют согласно установленным нормативно-техническими документами или требованиям Заявителя по предельным значениям ширины $(e)$ и высоты $(g)$ сечения выпуклости сварного шва с учетом физико-механического свойства расплавленного материала сварного шва решением интегродифференциального уравнения:

$$
\frac{d \tau}{d l}=-k_{0}+\frac{\cos \delta}{a_{k}^{2}} \int_{0}^{l} \operatorname{Sin} \tau d \lambda,
$$

где $l$ - расстояние от начальной точки эталонной кривой до точки расчета, измеренное вдоль кривой; значение $l$ изменяются от 0 до $L$;

$L$ - длина эталонной кривой сварного шва;

$d l$ - шаг дифференцирования вдоль эталонной кривой;

$d \lambda$ - шаг интегрирования вдоль эталонной кривой;

$\tau(l)$ - угол наклона касательной эталонной кривой к горизонту, в расчетной точке;

$k_{0}$ - кривизна эталонной кривой сварного шва в начальной точке, определяемая в ходе решения уравнения;

$a_{k}$ - физико-механическое свойство расплавленного материала сварного шва - капиллярная постоянная;

$\delta$ - угол продольного наклона сварного шва;

$\varphi$ - угол поперечного наклона сварного шва;

$Z(x)$ - уравнение, описывающее эталонные кривые сварного шва.

$$
\left.\begin{array}{c}
x=\int_{0}^{l}(\operatorname{Cos} \varphi \operatorname{Cos} \tau+\operatorname{Sin} \varphi \operatorname{Sin} \tau) d \lambda \\
Z=\int_{0}^{l}(-\operatorname{Sin} \varphi \operatorname{Cos} \tau+\operatorname{Cos} \varphi \operatorname{Sin} \tau) d \lambda
\end{array}\right\} 0 \leq l \leq L .
$$


Площадь эталона рассчитывают по формуле:

$$
S^{\ni}=\int_{0}^{e_{\max }}\left[Z^{\Im_{\max }}(x)-Z^{\Im_{\min }}(x)\right] d x .
$$

Применение технологии 3DLD позволяет:

- по форме поверхности сварного шва объективно оценивать практические навыки сварщика при сварке контрольных сварных соединений в процессе обучения, при сварке реальных сварных конструкций и при сравнении результатов во время соревнований сварщиков;

- визуально оценивать и измерять дефекты формы поверхности сварного шва;

- создать систему профессиональной оценки квалификации сварщиков в течение всей его профессиональной деятельности;

- значительно ускорить процесс проведения оценки сварных швов.

Преимуществом технологии 3DLD является еe объективность, мобильность, сравнительно невысокая стоимость и отсутствие необходимых расходных материалов.

Применение данной технологии позволяет оценивать стыковые контрольные сварные соединения (КСС) труб, стыковых и угловых пластин. Соответствие индекса квалификации сварщика, определенному по технологии 3DLD разряду сварщика по ЕТКС и профессиональному стандарту «Сварщик», представлено в Правилах «Системы добровольной сертификации квалификации сварщиков при сварке плавлением» РОСС RU.31452.04ИГC0 (таблица 1). 
Таблица 1. Таблица соответствия ИКС разрядам и уровню квалификации сварщика

\begin{tabular}{|c|c|c|}
\hline $\begin{array}{c}\text { Уровень квалификации сварщика } \\
\text { по профессиональному стандарту «Сварщик» }\end{array}$ & $\begin{array}{c}\text { Разряд сварщика } \\
\text { по ЕТКС }\end{array}$ & $\begin{array}{c}\text { ИКС, } \\
\text { баллы }\end{array}$ \\
\hline \multirow{2}{*}{2 (сварщик) } & 2 & $51-60$ \\
\hline \multirow{2}{*}{3 (сварщик) } & 3 & $61-70$ \\
\hline \multirow{2yy}{*}{4 (сварщик) } & 4 & $71-80$ \\
\hline \multirow{2}{*}{$\begin{array}{c}\text { п (сварщик бригадир, сварщик } \\
\text { производственного обучения) }\end{array}$} & 5 & $81-90$ \\
\hline
\end{tabular}

\section{Выводы}

Оценка квалификации сварщика в настоящее время базируется на субъективизме экспертов, принимающих решение о присвоении сварщику квалификационного уровня (разряда) при применении различных методов неразрушающего контроля сварных соединений. Существующие методы контроля, такие как ВИК, УЗК, РК и т.д., достаточно сложные, дорогостоящие и не определяют квалификационный уровень сварщика, а оценивают качество сварного шва по принципу «годен - не годен» и зависят от норм дефектности для различных отраслей промышленности.

Оценка качества выполненных контрольных сварных соединений с применением технологии 3DLD дает максимально объективный уровень подготовки специалиста и его возможности в профессиональном плане.

Качественная и объективная оценка квалификации сварщика позволит точнее отразить уровень подготовки специалистов, а также исключить субъективизм при проведении входного контроля сварщиков при допуске их на строительство опасных производственных объектов, что в дальнейшем приведет к снижению риска возникновения различных техногенных аварий. 


\section{Список используемых источников}

1. Лукьянов В.Ф., Кузьменко И.В. Методика обучения экспертной системы оценки стабильности работы сварщика // Вестник Донского государственного технического университета. 2014. Т. 14. № 4 (79). С. 117 124. DOI: $10.12737 / 6899$.

2. Как получить удостоверение сварщика в Москве // YouTube. 08.12.2019. URL: https://www.youtube.com/watch? $\mathrm{v}=\mathrm{KxK}$ Kexy4hiK8\&feature= youtu.be (дата обращения: 23.10.2020).

3. Панков В.В., Букин В.М., Панков С.В., Богородский И.Г. Оценка квалификации сварщика с применением цифровых технологий // Сварка и диагностика. 2012. № 6. С. 54-58.

4. Официальный сайт 3DLD. URL: http://3dld.ru/ (дата обращения: 29.09.2020).

5. Методика оценки качества сварного шва с применением новейшей технологии 3DLD // СтанкоЦентр «Талви». 06.04.2018. URL: http://talvi.ru/news/post/metodika-otsenki-kachestva-svarnogo-shva-sprimeneniyem-noveyshey-tekhnologii-3dld (дата обращения: 29.09.2020).

6. Букин В.М., Панков С.В., Панков В.В. Оценка качества сварочных электродов методом 3D лазерной диагностики поверхности сварного шва // Сварка и диагностика. 2014. № 1. С. 38-40.

7. Пат. 2550979 РФ, МПК В 23 К 31/12. Способ контроля качества поверхности сварного шва / В.В. Панков, В.М. Букин, С.В. Панков, И.Г. Богородский. 2013104776/02, Заявлено 04.02.2013; Опубл. 10.08.2014. Бюл. 2.

8. Пат. 2550673 РФ, МПК В 23 К 37/00. Устройство для оценки качества сварного шва / В.В. Панков, В.М. Букин, С.В. Панков, П.А. Крючков. 2013113394/02, Заявлено 25.03.2013; Опубл. 27.09.2014. Бюл. 27.

9. Пат. 2644617 РФ, МПК G 01 В 11/30. Мобильный сканер для определения качества поверхности сварного шва / В.В. Панков, В.М. Букин, С.В. Панков. 2016125509, Заявлено 21.06.2016; Опубл. 13.02.2018. Бюл. 5. 


\section{References}

1. Lukyanov V.F., Kuzmenko I.V. Metodika obucheniya ekspertnoi sistemy otsenki stabil'nosti raboty svarshchika [Expert System Training Technique to Evaluate Welder's Job Stability]. Vestnik Donskogo gosudarstvennogo tekhnicheskogo universiteta - Vestnik of Don State Technical University, 2014, Vol. 14, No. 4 (79), pp. 117-124. DOI: 10.12737/6899. [in Russian].

2. Kak poluchit' udostoverenie svarshchika v Moskve [How to Get a Welder's Certificate in Moscow]. YouTube. 08.12.2019. Available at: https://www.youtube.com/watch?v=KxKcxy4hiK8\&feature=youtu.be (accessed 23.10.2020). [in Russian].

3. Pankov V.V., Bukin V.M., Pankov S.V., Bogorodskii I.G. Otsenka kvalifikatsii svarshchika $\mathrm{s}$ primeneniem tsifrovykh tekhnologii [Welder Qualification Assessment using Digital Technologies]. Svarka i diagnostika Welding and Diagnostics, 2012, No. 6, pp. 54-58. [in Russian].

4. Official Website of 3DLD. Available at: http://3dld.ru/ (accessed 29.09.2020). [in Russian].

5. Metodika otsenki kachestva svarnogo shva s primeneniem noveishei tekhnologii 3DLD [Methodology for Assessing the Quality of a Welded Seam using the Latest 3DLD Technology]. StankoTsentr «Talvi». 06.04.2018. Available at: http://talvi.ru/news/post/metodika-otsenki-kachestva-svarnogoshva-s-primeneniyem-noveyshey-tekhnologii-3dld (accessed 29.09.2020). [in Russian].

6. Bukin V.M., Pankov S.V., Pankov V.V. Otsenka kachestva svarochnykh elektrodov metodom 3D lazernoi diagnostiki poverkhnosti svarnogo shva [Quality Assessment of Welding Electrodes using 3D Laser Diagnostics of the Weld Surface]. Svarka i diagnostika - Welding and Diagnostics, 2014, No. 1, pp. 38-40. [in Russian].

7. Pankov V.V., Bukin V.M., Pankov S.V., Bogorodskii I.G. Sposob kontrolya kachestva poverkhnosti svarnogo shva [Method for Controlling the Surface Quality of a Weld]. Patent RF, No. 2550979, 2014. [in Russian]. 
8. Pankov V.V., Bukin V.M., Pankov S.V., Kryuchkov P.A. Ustroistvo dlya otsenki kachestva svarnogo shva [Device for Assessing the Quality of the Weld]. Patent RF, No. 2550673, 2014. [in Russian].

9. Pankov V.V., Pankov S.V., Bukin V.M. Mobil'nyi skaner dlya opredeleniya kachestva poverkhnosti svarnogo shva [Mobile Scanner for Determining the Surface Quality of the Weld]. Patent RF, No. 2644617, 2018. [in Russian].

\section{Сведения об авторах}

\section{About the authors}

Файрушин Айрат Миннуллович, канд. техн. наук, доцент, заведующий кафедрой «Оборудование и технологии сварки и контроля», УГНТУ, г. Уфа, Российская Федерация

Airat M. Fairushin, Candidate of Engineering Sciences, Associate Professor, Head of Equipment and Technologies for Welding and Control Department, USPTU, Ufa, Russian Federation

e-mail: otsk@rusoil.net

Марченко Илья Алексеевич, студент кафедры «Оборудование и технологии сварки и контроля», УГНТУ, г. Уфа, Российская Федерация

Ilya A. Marchenko, Student of Equipment and Technologies for Welding and Control Department, USPTU, Ufa, Russian Federation

e-mail: ilya_marchenko_1976@mail.ru

Хазиев Виктор Сергеевич, эксперт Союза WorldSkills Russia, магистрант кафедры «Оборудование и технологии сварки и контроля», УГНТУ, г. Уфа, Российская Федерация

Viktor S. Khaziev, Expert of the WorldSkills Russia Union, Undergraduate Student of Equipment and Technologies for Welding and Control Department, USPTU, Ufa, Russian Federation

e-mail: victor.khaziev@mail.ru 